Mariarosa Carta*, Luciana Bragagnolo, Andrea Tramarin, Elena Barzon, Anna Cappelletti, Michela Pascarella, Lauretta Forner, Maria Grazia Meneghini, Chiara Tripodi, Mario Rassu and Davide Giavarina

\title{
Anti SARS-CoV-2 antibodies monitoring in a group of residents in a long term care facility during COVID-19 pandemic peak
}

https://doi.org/10.1515/dx-2020-0094

Received July 8, 2020; accepted July 8, 2020; published online August 22, 2020

\section{Abstract}

Objectives: Clinical laboratories plays a key role in screening, diagnosis and containment of the Coronavirus 2019 infection epidemic. The etiological diagnosis presupposes the isolation of virus genetic material in the patient's biological sample but laboratory diagnostics also make use of searching possibility for immunoglobulin (Ig)G, IgM classes antibodies. The characteristics of the antibody response are not yet completely clear.

Methods: This study describes a serological monitoring of subjects, elderly nursing care residence guests, interested by a very large infection outbreak. After first nasopharyngeal swab, all the positive subjects (43) were monitored for the persistence of the virus infection through nasopharyngeal swab after 20 days (16-24), 32 days (28-36) and after 49 days (47-50). At the same time, during the second (day 32) and third (day 49) follow up, all the guests were investigated for IgM and IgG anti SARS-CoV-2 antibodies, by using a quantitative chemiluminescence method.

Results: Thirty two days after performing the first diagnostic swab, 39 of 43 patients (90\%) had IgG higher than the cut off value. After 49 days the four patients with negative IgG were still negative. The comparison of the levels of IgG-Ab between the controls shows a significant decrease in concentrations $(-10 \%)$.

*Corresponding author: Mariarosa Carta, Medicina di Laboratorio, AULSS 8 Berica, Viale Rodolfi, Vicenza, Italy, Phone: +39 0444 752486, Fax: +39 0444 752501,

E-mail: mariarosa.carta@aulss8.veneto.it Luciana Bragagnolo, Michela Pascarella and Mario Rassu: Microbiologia, AULSS 8 Berica, Vicenza, Italy Andrea Tramarin: Cure Primarie-Distretto Est, AULSS 8 Berica, Vicenza, Italy

Elena Barzon, Anna Cappelletti, Lauretta Forner, Maria Grazia Meneghini, Chiara Tripodi and Davide Giavarina, Medicina di Laboratorio, AULSS 8 Berica, Vicenza, Italy. https:// orcid.org/0000-0001-9589-178X (D. Giavarina)
Conclusions: Our study confirms that in most patients affected by COVID-19 there is a typical antibody response with IgG-Ab present in $90 \%$ of nursing care COVID-19 positive residence guests. For IgM-Ab only $23 \%$ of tested subjects were positive on the 32nd and 49th day of illness, always in parallel with the IgG-Ab positivity.

Keywords: COVID-19; immunoglobulin (Ig)G; IgM.

\section{Introduction}

On January 19, 2020, the World Health Organization (WHO) declared COVID-19 disease an international public health emergency. The epidemic soon affected Italy and the Italian Council of Ministers declared the state of emergency on the entire national territory 11 days later.

The pathogen of the epidemic has soon been identified as a new Coronavirus, never previously identified in humans. This virus, later defined as SARS-CoV-2 [1], is an RNA virus belonging to the betacoronavirus genus, with a pericapsid, round or oval in shape and often polymorphic, with a diameter of $60140 \mathrm{~nm}$. Its genetic characteristics significantly differ from previous severe acute respiratory syndrome Coronavirus (SARSr CoV) as well as from the Middle East Respiratory Syndrome Coronavirus (MERS-CoV) and current research shows a homology of more than $85 \%$ with bat SARS like Coronavirus (bat SL CoVZC45) [2].

Infected people mainly transmit the SARS-CoV-2 virus through breath droplets, but the virus can be also transmitted by contact.

The symptoms are very varied, and in the initial stage of the disease, it is mainly characterized by fever, cough, dyspnoea, asthenia and myalgia. Subsequently, it may evolve into the acute respiratory distress syndrome (ARDS) [2]. In most cases, individuals infected with SARS-CoV-2 are asymptomatic or exhibit mild symptoms, most likely due to a good "innate" response that activates defence mechanisms involving $\mathrm{T}$ lymphocytes and natural killers with antiviral action, and through an interferon induction [3]. 
Unfortunately, in $20 \%$ of subjects the disease can present much more serious forms, particularly in older people or subjects with variously compromised health conditions, for cardiovascular and lung problems, diabetics, hypertension, obesity, pulmonary obstructive chronic lung disease (both COPD and emphysema), fibrosis, asthma and interstitial lung disease [4]. In most of these cases the pathology involves mainly - though not exclusively - the lung, with an interstitial pneumonia, which can lead to ARDS and even death.

Clinical laboratories play a key role in screening, diagnosis and containment of the Coronavirus 2019 infection epidemic (COVID-19). In fact, the presence of asymptomatic but infected and contagious individuals jeopardizes the possibility of a diagnostics based only on the clinic signs and symptoms [5].

The etiological diagnosis of COVID-19 presupposes the isolation of virus genetic material (RNA) in the patient's biological samples. According to the WHO and the Italian Health Superior Institute (ISS), the reference method is the detection of viral RNA using reverse transcriptase polymerase chain reaction technique (RT-PCR), on oral and nose/ pharyngeal swabs [6]. Laboratory diagnostics also make use of searching possibility for IgG, IgM classes antibodies and, more recently, IgA [7], aimed against SARS-CoV-2 antigens.

It is generally believed that the role of antibody research is limited to epidemiological studies of seroprevalence, in screening searching for potential plasma donors, during convalescence, in the effectiveness evaluation of a vaccination. It can also be used as an aid in COVID-19 diagnosis on patients RT-PCR negative for SARS-CoV-2 who subsequently get the disease, without the possibility to analyse a deep respiratory tract sample [8].

Until now, the use of anti-SARS-CoV-2 antibody detection is not yet well defined and substantially limited. The antibodies measurement should not be used to guide clinical decisions, neither regarding the use or not of personal protective equipment or social distancing, or as a screening for access to direct biomolecular diagnosis. Moreover, the characteristics of the antibody response are not yet completely clear: different patterns are described in different cases $[9,10]$, especially as regards IgM. This may be due to behaviour of the virus, which in a recent editorial has been defined as "bizarre" [10], but this variability also depends on the use of antibodies measurement methods based on different principles, as well as directed towards different epitopes and antigens.

The problem of analytical methods seems particularly critical. In fact, the antibodies assays is available both with rapid qualitative methods (POCT) based on immunochromatography principles of and with quantitative methods, as chemiluminescence immunoassays (CLIA, ECLIA) or enzyme-linked immunosorbent assay (ELISA).

In the next close future, studying the serological response and the positive antibody kinetics could be useful to understand whether these tests will help the care of single individual or the surveillance of groups at risk, or could be even used for screening or general surveillance purposes.

This study describes a serological monitoring of the residents in a long term care facility (LTCF), interested by a very large infection outbreak. On March 2020, a SARS-Cov-2 infection affected a LTCF with 66 guests, in the province of Vicenza district, a little town in eastern Italy. After the first positive patient, who died a few days after the diagnosis, the infection hit most of the guests of the facility. All guests were monitored for a period of two months from the outbreak, using a CLIA assay that uses monoclonal antibodies directed both against the spike protein of SARS-CoV2 , and against the nucleocapsid region of the virus.

\section{Materials and methods}

The study involved all the guests (symptomatic and asymptomatic) of a LTCF. After the first case of infection rapidly resulted in the death for COVID-19 of the elder guest, all the resident subjects were studied for the Coronavirus infection by using a real time polymerase chain reaction method (RT-PCR), within a period March 29 and April 22, 2020. The subjects included in the study were 65 ( 13 males and 52 females, average age 82 years (56-97), 26 of them not self-sufficient.

Virus detection was performed on a sample collected using oropharyngeal and nasopharyngeal swab (Copan Universal Transport Medium, UTM ${ }^{\circledast}$ System, Murrieta, CA, USA). Qualitative detection of nucleic acids from SARS-CoV-2 was performed on Cobas 6800 RT-PCR System (Roche Diagnostics GmbH, Mannheim, Germany). The system is based on fully automatic sample preparation (nucleic acid extraction and purification) followed by a PCR amplification and detection. Dedicated software automatically assigns the results for each individual test.

Selective amplification of target nucleic acid from the sample is achieved by the use of target-specific forward and reverse primers for ORF1/a non-structural region that is unique to SARS-CoV-2. Additionally, a conserved region in the structural protein envelope E-gene was chosen for pan-Sarbecovirus detection. When two gene targets are found both positive, or even if only one target is found, but the patient has characteristic symptoms, the test is considered positive.

After first oropharyngeal/nose swab, all the subjects were monitored for the persistence of the virus infection through subsequent swabs after 20 days (16-24), 32 days (28-36) and, only in still positive cases, after 49 days (47-50). At the same time, during the second (day 32) and third (day 49) tests, all the residents were investigated for IgM and IgG anti SARS-CoV-2 antibodies, using a quantitative chemiluminescence method. Subjects with RT-PCR negative results underwent a single measurement of antibody production, at the end of the study, at the same time with the execution of an oropharyngeal/nose swab. The serum samples, collected in a test tube 
with coagulation activator and without separator gel (Vacutest, Kima, Arzegrande, PD, Italy) were inactivated at $56{ }^{\circ} \mathrm{C}$ for $30 \mathrm{~min}$, as recommended by the producer. After centrifugation, samples were measured on Maglumi 800 instrumentation, by a chemiluminescence immunoassay with capture antibodies, MAGLUMI 2019-nCoV IgG and IgM (CLIA) (Shenzhen New Industries Biomedical Engineering Co., SNIBE diagnostic, Shenzhen, China).

The samples were analyzed within $4 \mathrm{~h}$ of collection or stored in a refrigerator at $4^{\circ} \mathrm{C}$ until the following day, processed within $24 \mathrm{~h}$ from collection. The method is based on two different monoclonal antibodies, anti IgG and anti IgM, labelled with N-(aminobutyl)-N-(ethylisoluminol) (ABEI), which acts as a chemiluminescent reagent. The method was CE marked on February 28, 2020.

The antibody research results were evaluated according to the manufacturer's indications. IgG antibodies (IgG-Ab) were considered negative for concentrations lower than $0.90 \mathrm{AU} / \mathrm{mL}$, positive if greater than or equal to $1.10 \mathrm{AU} / \mathrm{mL}$, with a grey-zone between 0.90 and 1.10 . For IgM antibodies (IgM-Ab), values greater than or equal to $1.00 \mathrm{AU} /$ $\mathrm{mL}$ were considered positive, with no grey-zone between positive and negative.

Statistical significance for the differences was calculated with the Wilcoxon test and Mann-Whitney test (MedCalc v.192.6).

The study was approved by the Ethics Committee and was done in compliance with the World Medical Association Declaration of Helsinki.

\section{Results}

Among 65 guests residing in the LTCF, 54 resulted positive for the COVID-19 virus on the first swab, but 11 patients rapidly evolved to death (10 in the first days after diagnosis and 1 after a long hospital stay). Monitoring therefore involved 43 patients ( 8 males and 35 females, average age 81 years (56-97), 24 self-sufficient and 19 non-selfsufficient guests, 14 with psychiatric pathology. Eleven subjects did not develop the infection (permanently negative swabs) in the following 60 days.

After 49 days all 43 patients (100\%), previously positive, were negative for RT-PCR for SARS-CoV-2. Twenty-two patients (51\%) were already negative at the first control after 20 days, 32 patients (74\%) at the second control, after 32 days (Table 1$)$.

\section{SARS-CoV-2 IgG antibodies}

Thirty-two days after the first laboratory-confirmed case, 39 of 43 patients $(90 \%)$ had IgG higher than the cut off value $(>1.1 \mathrm{AU} / \mathrm{mL})$. Among four patients with negative IgG-Ab, two actually had RT-PCR for SARS-CoV-2 RNA still positive. IgG-Ab concentration were higher in the group of patients with RT-PCR swab test negative (median=23.0 AU/
Table 1: Timing of oropharyngeal/nose swab in 43 subjects affected by COVID-19.

\begin{tabular}{lrrrr}
\hline & $\begin{array}{r}\text { Diagnosis } \\
\text { (time 0) }\end{array}$ & $\begin{array}{r}\text { Control n.1 } \\
\text { (after } \\
\text { 20 days) }\end{array}$ & $\begin{array}{r}\text { Control n.2 } \\
\text { (after } \\
\text { 32 days) }\end{array}$ & $\begin{array}{r}\text { Control n.3 } \\
\text { (after } \\
\text { 49 days) }\end{array}$ \\
\hline $\begin{array}{c}\text { RT-PCR } \\
\text { positive }\end{array}$ & 43 & 21 & 11 & 0 \\
$\begin{array}{c}\text { RT-PCR } \\
\text { negative }\end{array}$ & 0 & 22 & 32 & 43 \\
\hline
\end{tabular}

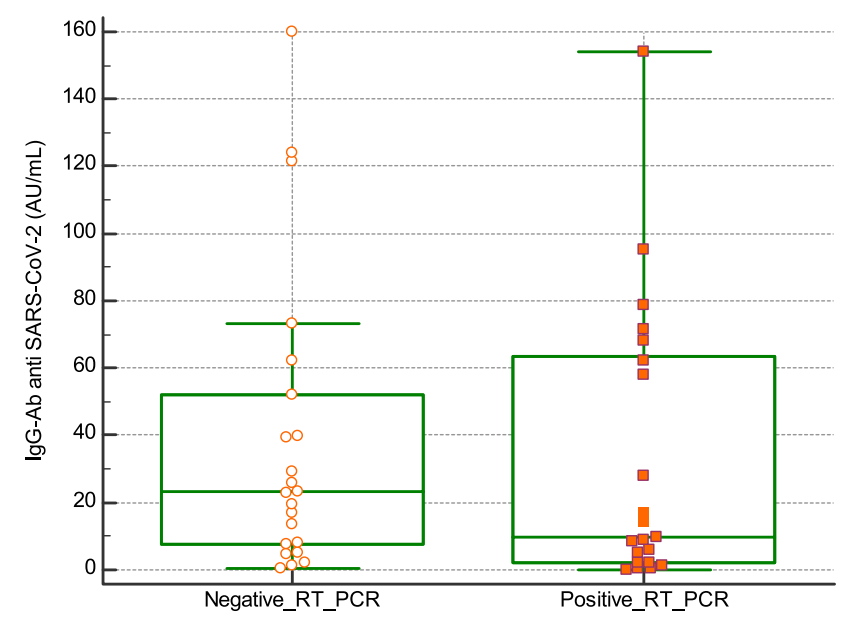

Figure 1: Anti Sars-CoV-2 IgG concentrations in patient affected by COVID-19, after 32 days from the diagnosis, compared for current negative and positive RT-PCR test (oropharingeal/nose swab).

$\mathrm{mL}$ ) than in those positive one (median=9.9 AU/L) but the difference was not statistically significant (Mann-Whitney test, $\mathrm{p}=0.39$ ) (Figure 1).

After 49 days from the diagnosis, 39 out of 43 patients (90\%) confirmed IgG higher than the cut off. The four patients with negative IgG were the same who were negative even at the first control. In all these four patients the swab test had now negativized.

Comparing the IgG Ab anti Sars-CoV-2 concentrations measured in the second control with the concentrations measured in the first one, the difference is statistically significant (Wilcoxon test for paired data, $\mathrm{p}<0.001$; median value 17.0 and $12.1 \mathrm{AU} / \mathrm{mL}$ in the first and second control, respectively) (Figure 2). Mean difference between the first and the second control was $10 \%$.

The Anti Sars-CoV-2 IgG-Ab test in the 11 subjects with persistently negative RT-PCR identified one patient with elevated IgG-Ab (27.0 AU/mL), while the other 10 had values below the cut off. 


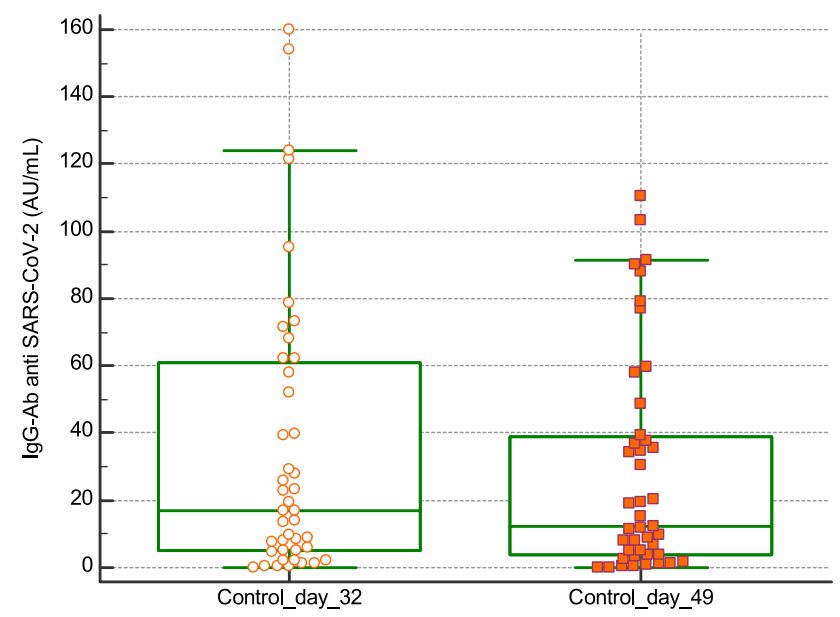

Figure 2: Anti Sars-CoV-2 IgG concentrations comparison between first (day 20) and second (day 49) serological control.

\section{SARS-CoV-2 IgM antibodies}

Thirty-two days after the first laboratory-confirmed case, 10 of 43 patients $(23 \%)$ had IgM-Ab higher than the cut off value $(>1.0 \mathrm{AU} / \mathrm{mL})$. Among these 10 patients, seven were still positive to the swab test. The distribution of concentration in the patients with negative swab showed a median value of $0.6 \mathrm{UA} / \mathrm{mL}(0.4-2.9)$, while in patients with positive swab the median concentration value was $0.7 \mathrm{UA} / \mathrm{mL}$ (0.4-5.6) (Figure 3).

At the second control (day 49), all the 10 previous positive patients for IgM antibodies were confirmed positive, while all the negative remained negative. The positive subjects for IgM-Ab had also IgG-Ab levels over the cut-off.

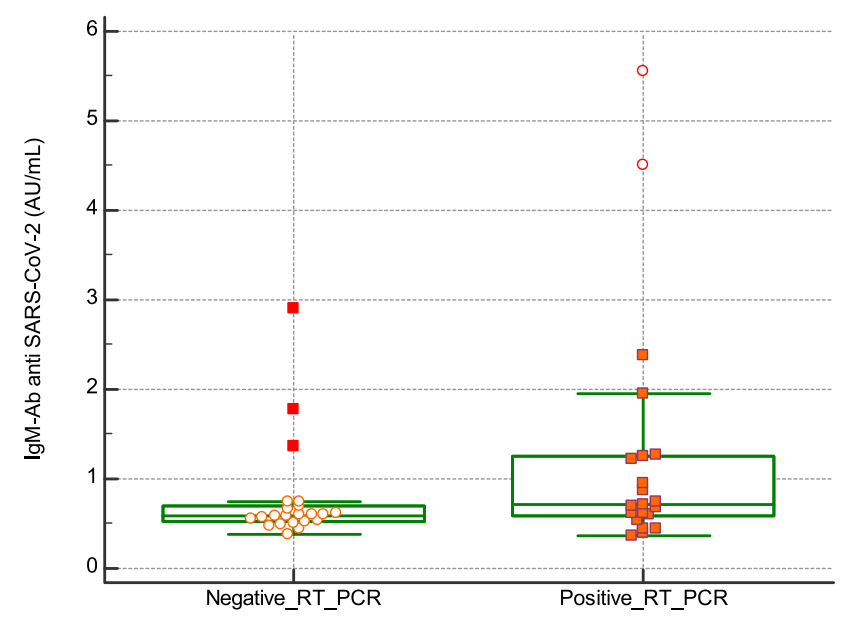

Figure 3: Anti Sars-CoV-2 IgM concentrations in patient affected by COVID-19, after 32 days from the diagnosis, compared for current negative and positive (day 20) RT-PCR test (oropharingeal/nose swab).

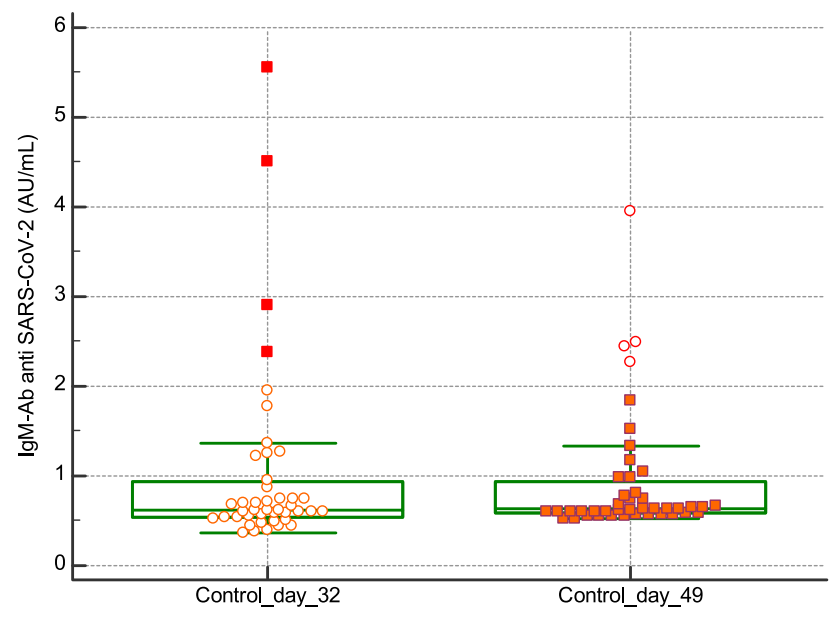

Figure 4: Anti Sars-CoV-2 IgM concentrations comparison between first (day 20) and second (day 49) serological control.

The median difference between the IgM Ab levels in the first control and in the second was not statistically significant (median 0.62 vs. 0.63; Wilcoxon test for paired data $\mathrm{p}=$ n.s.) (Figure 4).

The anti Sars-CoV-2 IgM-Ab test in the 11 subjects with persistently negative RT-PCR remain negative in all the controls.

\section{Discussion}

In viral infectious diseases, direct diagnosis and serological diagnosis (indirect) give us two completely different information. The first test informs about the incidence, that is, indicates new cases of infection and tells us when the virus is present; on the contrary, the antibody test provides a prevalence value, that is, it tells how many people have come into contact with the virus, for how long and if and how specific the immune response could be.

In the diagnosis of SARS-CoV-2 infection, molecular analysis is the gold standard, to the point that the confirmed case occurs with the patient positive for RT-PCR analysis, even in the absence of clinical symptoms [5]. However, the serological diagnosis is under constant evaluation and arouses great interest, even in the acute phase of the SARS-CoV-2 epidemic, for many reasons.

Firstly, the biomolecular detection methods are based on the presence of the viral genome, which have to be amplified. A first limit can be linked to the viral load in the sample collection site but also to the lack of the viral replication time window, which can provide false negative results [12]. Furthermore, an imperfect collection of the sample can produce false negative results, due to the 
presence of not enough virus in the swab sample [6]. Therefore, the evaluation of a possible contribution of indirect serological diagnosis is also interesting, even in the acute phase of the epidemic, alongside the detection for the viral genome, at least in certain settings. However, it would be helpful to better know the kinetics and the behaviour over time of the anti-SARS-CoV-2 antibodies. In fact, if it has been well demonstrated that immunoglobulins of IgG class generally arise from 10 to 18 days from the onset of symptoms and they are present after four weeks from the infection [11], knowledge relating to the behaviour of IgM-Ab is most controversial.

Guo et al. [12] report the appearance of IgM-Ab at an early stage (three days after the onset of symptoms) in 85\% of patients diagnosed or suspected to be COVID-19 affected, and the appearance of IgG-Ab in $78 \%$, after a median period of 14 days. On the other hand, To et al. [4] report a case of IgG-Ab detectable before the appearance of IgM-Ab, while Long et al. [13] describe different seroconversion modes: synchronous (simultaneous development of IgG and IgM), IgM first, then IgG-Ab detection, or finally IgG first than IgM-Ab.

Our study confirms that in most patients affected by COVID-19 there is a typical antibody response to acute infection. On the thirty-second day from the Sars-CoV-2 infection diagnosis, IgG-Ab were present in almost all (90\%) of nursing care COVID positive residence guests, with slightly higher values in patients with swab already become negative. However, it is worth noting that four patients did not show the presence of IgG-Ab neither after 32 days nor in the second control (49 days after diagnosis), albeit with negative swab.

The comparison of IgG-Ab levels between the first and the second control shows a significant decrease in concentrations $(-10 \%)$. In the control group (11 nursing home guests with persistently negative swabs), only one patient had high IgG values with negative IgM. A previous paucisymptomatic infection can be hypothesized, which occurred before the epidemic outbreak in the LTCF.

On the other hand, the presence of IgM concerned only $23 \%$ of the patients and, also in this case, repeating the control after 20 days had no practical effects: all the IgM positive patients in the first control remain positive also in the second one. At the second control IgM-Ab concentrations were lower than at the first, but the median differences were not statistically significant. Low IgM-Ab sensitivity could refer to its kinetic. IgM-Ab concentration levels reach the peak around the 12th day from the infection, while starting from the 18th day they start to fall [14]. Our first determination was made on the 32nd day. However, the IgM values in our study, while showing a decline, remained above the cut off until day 49 . Other studies [15] have confirmed that the presence of IgM-Ab is confirmed in less than $50 \%$ of SARS-CoV-2 infection.

This study was carried out by using an immunochemiluminescence method, with the European Conformity certification. The manufacturer declared both sensitivity and specificity were greater than $95 \%$. Anyway, for IgM-Ab, our data seem unable to confirm such a high sensitivity.

The main limitation of this study is the delay in performing the antibody research since the COVID-19 diagnosis. About IgM-Ab, researching IgM-Ab within 12 days is proposed [14]. However, the positivity of IgG-Ab, already present after about 32 days in $90 \%$ of cases, suggests a possible role of the serological examination to document the occurrence of SARS-CoV-2 infection.

Considering that the IgG-Ab determinations carried out 49 days after the COVID-19 diagnosis provided the same percentage of positive results than testing at day 32, without any difference between the first and the second measurement, the test sensitivity turns out to be $90 \%$. Repeating the search for IgG-Ab after a while does not seem to offer anything more, although the significant decrease in IgG concentrations, which occurred in just 50 days, seems to indicate a rather dynamic kinetics of IgG.

As regards to IgM-Ab, also in this case the repetition of the test after some time did not change what was seen in the first control. However, only $23 \%$ of subjects tested positive on the 32nd and 49th day of illness, always in parallel with the IgG-Ab positivity. Consequently, the role of serological diagnostics seems currently confined to the IgG-Ab search only, in order to identify patients who have come into contact with the virus, rather than for screening and diagnosis purposes.

In conclusion, these data seem to confirm that immunological seroconversion, detectable through the appearance of IgG class SARS-CoV-2 antibodies, occurs in the great majority of cases of infection, at least in elderly populations. The seroconversion appears complete already on the 32nd day and further determinations do not change the prevalence. On the other hand, the contribution of the measure of IgM-Ab is poor. The study therefore confirms previous data from recent literature [16], suggesting that serological surveillance can find its own field of application especially in the later stages of infection (when the virus has already been eliminated), or in the surveillance of asymptomatic people exposed to infection, in surveillance epidemiological studies and finally in communities, to define and control the wideness of viral spread. 
Research funding: None declared.

Author contributions: All authors have accepted responsibility for the entire content of this manuscript and approved its submission.

Competing interests: Authors state no conflict of interest. Informed consent: Informed consent was obtained from all individuals included in this study.

Ethical approval: The study was approved by the Ethics Committee and was done in compliance with the World Medical Association Declaration of Helsinki.

\section{References}

1. Coronaviridae Study Group of the International Committee on Taxonomy of Viruses. The species Severe acute respiratory syndrome-related coronavirus: classifying 2019-nCoV and naming it SARS-CoV-2. Nat Microbiol 2020;5:536-44.

2. Jiang F, Deng L, Zhang L, Cai Y, Cheung CW, Xia Z. Review of the clinical characteristics of coronavirus disease 2019 (COVID-19). J Gen Int Med 2020;35:1545-9.

3. Chen Y, Liu Q, Guo D. Emerging coronaviruses: genome structure, replication, and pathogenesis. J Med Virol 2020;92: 418-23.

4. Maragakis L. Coronavirus and COVID-19: who is at higher risk?. Johns Hopkins Med 2020. Available from: https://www. hopkinsmedicine.org/health/conditions-and-diseases/ coronavirus/coronavirus-and-covid19-who-is-at-higher-risk.

5. Plebani M. Il valore della medicina di laboratorio nella pandemia da SARS-CoV-2. Biochim Clin 2020;1-5. https://doi.org/10.19186/ BC_2020.053 [Epub ahead of print].

6. Lippi G, Simundic AM, Plebani M. Potential preanalytical and analytical vulnerabilities in the laboratory diagnosis of coronavirus disease 2019 (COVID-19). Clin Chem Lab Med 2020;58: 1070-6.
7. Padoan A, Sciacovelli L, Basso D, Negrini D, Zuin S, Cosma C, et al. IgA-Ab response to spike glycoprotein of SARS-CoV-2 in patients with COVID-19: a longitudinal study. Clin Chim Acta 2020;507: 164-6.

8. Theel ES, Slev P, Wheeler S, Couturier MR, Wong SJ, Kadkhoda K. The role of antibody testing for SARS-CoV-2: is there one?. J Clin Microbiol 2020;JCM.00797-20. https://doi.org/10.1128/JCM. 00797-20 [Epub ahead of print].

9. To KK, Tsang OT, Leung WS, Tam AR, Wu TC, Lung DC, et al. Temporal profiles of viral load in posterior oropharyngeal saliva samples and serum antibody responses during infection by SARS-CoV-2: an observational cohort study. Lancet Infect Dis 2020;20:565-74.

10. Sethuraman N, Jeremiah SS, Ryo A. Interpreting diagnostic tests for SARS-CoV-2. JAMA 2020;323:2249-51.

11. Lippi G, Salvagno GL, Mattiuzzi C. Guida sintetica alla diagnostica della malattia da coronavirus 2019 (COVID-19). Biochim Clin 2020;1-6. https://doi.org/10.19186/BC-2020.052 [Epub ahead of print].

12. Guo L, Ren L, Yang S, Xiao M, Chang D, Yang F, et al. Profiling early humoral response to diagnose novel coronavirus disease (COVID-19). Clin Infect Dis 2020;1-8. https://doi.org/10.1093/cid/ciaa310 [Epub ahead of print].

13. Long QX, Liu BZ, Deng HJ, Wu GC, Deng K, Chen YK, et al. Antibody responses to SARS-CoV-2 in patients with COVID-19. Nat Med 2020;26:845-8.

14. Padoan A, Cosma C, Sciacovelli L, Faggian D, Plebani M. Analytical performances of a chemiluminescence immunoassay for SARS-CoV-2 IgM/IgG and antibody kinetics. Clin Chem Lab Med 2020;58:1081-8.

15. Jin Y, Wang M, Zuo Z, Fan C, Ye F, Cai Z, et al. Diagnostic value and dynamic variance of serum antibody in coronavirus disease 2019. Int J Infect Dis 2020;94:49-52.

16. Plebani M, Padoan A, Negrini D, Carpinteri B, Sciacovelli L. Diagnostic performances and thresholds: the key to harmonization in serological SARS-CoV-2 assays?. Clin Chim Acta 2020;509:1-7. 